Check for updates

Cite this: RSC Adv., 2018, 8, 4214

Received 5th September 2017

Accepted 3rd January 2018

DOI: $10.1039 / c 7 r a 09894 d$

rsc.li/rsc-advances

\section{Qualitatively and quantitatively characterizing water adsorption of a cellulose nanofiber film using micro-FTIR spectroscopy}

\begin{abstract}
Xin Guo, (D) ${ }^{a}$ Liu Liu, ${ }^{a}$ Junjie Wu, ${ }^{a}$ Jia Fan ${ }^{b}$ and Yiqiang Wu ${ }^{* a}$
The efficient utilization of cellulose is very important for environmental protection and sustainability of resources. A cellulose derivative, cellulose nanofiber film, is a highly promising candidate for use in tissue engineering, electronics, and green packaging materials; however, water adsorption seriously impairs the properties of cellulose nanofiber film. In order to efficiently utilize this cellulose derivative, we need to qualitatively and quantitatively characterize its water adsorption. In situ micro-FTIR spectra of the cellulose nanofiber film were collected within a relative humidity $(\mathrm{RH})$ range of $0-94 \%$. The spectral information indicated effective adsorption sites for adsorbed water and identified three spectral regions closely associated with water absorption. Meanwhile, moisture contents of the cellulose nanofiber film were measured by dynamic vapor sorption as reference values. Using a partial least squares regression (PLS-R) method, the identified spectral regions closely associated with water absorption were used to construct a multivariate quantitative model to predict moisture contents of cellulose nanofiber film over the entire $\mathrm{RH}$ range. The predicted sorption isotherm confirmed the effectiveness of the micro-FTIR spectroscopy in quantitatively characterizing water adsorption of the cellulose nanofiber film at room temperature of $25^{\circ} \mathrm{C}$.
\end{abstract}

\section{Introduction}

Cellulose is a sustainable raw material that can be derived from agricultural residues. ${ }^{1-3}$ The efficient utilization of cellulose is very important for both protecting the environment and sustainably using natural resources. Cellulose nanofiber film, which is a novel cellulose derivative, has excellent physical, chemical, and biological properties, such as good thermal stability, high strength, low degradation, and non-toxicity, ${ }^{\mathbf{4}, 5}$ and it is a very promising candidate for tissue engineering, electronics, and green packaging materials. ${ }^{6,7}$ However, hydrophilic cellulose nanofiber film adsorbs water under hydrothermal conditions, which strongly affects its surface behavior and can lead to reliability problems. ${ }^{8,9}$ Consequently, the water adsorption mechanisms of cellulose nanofiber film need to be fully understood in order for this sustainable raw material to be efficiently utilized.

Water adsorption is a key property of cellulose materials, and it has been studied via a number of experimental approaches, such as quartz crystal microgravimetry, ${ }^{10}$ dynamic vapor sorption, ${ }^{\mathbf{1 1}, 12}$ dielectric relaxation spectroscopy, ${ }^{\mathbf{1 3}}$ Fourier transform infrared spectroscopy, ${ }^{\mathbf{1 4}}$ and nuclear magnetic resonance

${ }^{a}$ College of Materials Science and Engineering, Central South University of Forestry and Technology, Changsha 410004, China. E-mail:wuyq0506@126.com

${ }^{b}$ College of Life Science and Technology, Central South University of Forestry and Technology, Changsha 410004, China spectroscopy. ${ }^{15,16}$ Of these experimental approaches, dynamic vapor sorption (DVS) is an important quantitative method which has been widely used and has provided vast amounts of water sorption data. ${ }^{17-19}$ Using this method, sorption isotherm and sorption hysteresis of many cellulose materials including natural fibers, ${ }^{20,21}$ regenerated cellulose, ${ }^{22}$ microcrystalline cellulosic fibers, ${ }^{23}$ and wood powder, ${ }^{24}$ have been analyzed. As this method has been confirmed to be able to give highly reproducible sorption data of tested sample over a wide $\mathrm{RH}$ range in real time, ${ }^{25-27}$ it can be used as a reference method to quantitatively analyze the water adsorption. Meanwhile, Fourier transform infrared (FTIR) spectroscopy shows some distinct advantages because its spectral information allowed both qualitative and quantitative analyses of the water adsorption. In addition, micro-FTIR spectroscopy has recently been developed, and it has better sensitivity for the detection of water than conventional FTIR approaches. This is because micro-FTIR spectroscopy equipment contains an additional visible-light microscope that can be used to visualize the morphology and select observation area of micro-sized sample. ${ }^{28}$ As a result, micro-FTIR spectroscopy is believed to hold promise for the investigation of the water adsorption of nanocellulose materials such as cellulose nanofiber film.

Many interesting results about the water adsorption characteristics of cellulose materials have been obtained using FTIR spectroscopy. For example, Célino et al. ${ }^{14}$ confirmed that FTIR spectroscopy had the potential to qualitatively analyze the 
moisture absorption of natural fibers. Olsson et al. ${ }^{29}$ used FTIR spectroscopy to examine the water adsorption of cellulose materials, and determined that there was an important relationship between the weight gain due to moisture and increases in its $\mathrm{O}-\mathrm{H}$ stretching envelope. Haxaire et $a l^{30}$ used FTIR spectroscopy to determine whether the $\mathrm{OH}$ groups were bonded with water. Hofstetter et $a l^{31}$ investigated the interaction between cellulose and moisture via FTIR spectroscopy and showed the role that different hydrogen bonds played in water adsorption. Kachrimanis et $a .^{32}$ and Berthold et $a .^{33}$ both demonstrated that the chemical sites for water adsorption for partly carboxymethylated cellulose were $\mathrm{OH}$ and $\mathrm{C}=\mathrm{O}$ groups. Guo et $a .^{34}$ confirmed that carboxyl $\mathrm{C}=\mathrm{O}$ and $\mathrm{C}-\mathrm{O}$ groups as well as $\mathrm{OH}$ groups were active sites for water adsorption in wood specimen. These works given as examples in this paragraph are very important, as they have provided much FTIR spectral information for qualitatively characterizing water adsorption of cellulose materials. However, based on univariate analysis of this FTIR spectral information, water sorption isotherms of cellulose materials were not properly determined.

The aim of this work is the use of micro-FTIR spectroscopy as an experimental tool to qualitatively and quantitatively characterize water adsorption of nanocellulose materials (i.e., cellulose nanofiber film) at room temperature of $25{ }^{\circ} \mathrm{C}$. Firstly, we collected in situ micro-FTIR spectra of cellulose nanofiber film over a wide range of $\mathrm{RH}$ levels to indicate effective adsorption sites for adsorbed water and identify spectral regions affected by water adsorption. We also measured moisture contents of cellulose nanofiber film using DVS apparatus as reference values. Secondly, a multivariate model linking the measured moisture contents and the selected micro-FTIR spectral regions was developed using partialleast square regression (PLS-R) method. Finally, we estimated sorption isotherms using this constructed multivariate model.

\section{Materials and method}

\subsection{Sample preparation}

This paper focused on a typical specimen of nanocellulose material (i.e., cellulose nanofiber film), and the preparation of this cellulose nanofiber film was described clearly in earlier reports. ${ }^{35}$ In the present study, we used the same procedure. Once the film was obtained, it was covered by two pieces of cover slips and dried under atmospheric conditions. Before the spectral experiment, the film was dried a further time in nitrogen gas for at least $2 \mathrm{~h}$.

\subsection{Micro-FTIR spectroscopy apparatus}

Fig. 1 shows a diagram of the micro-FTIR spectroscopy apparatus. The main part of the apparatus was a micro-FTIR spectrometer (Nicolet IN 10T, Thermo Electron Scientific Instruments, Madison, WI, U.S.A.). As stated in Section 1, this spectrometer was equipped with one more microscope than was found in conventional FTIR spectrometers; in this microscope, two pathways for visible and IR beams were included; the visible beam came from a visible light source, and it could be used for

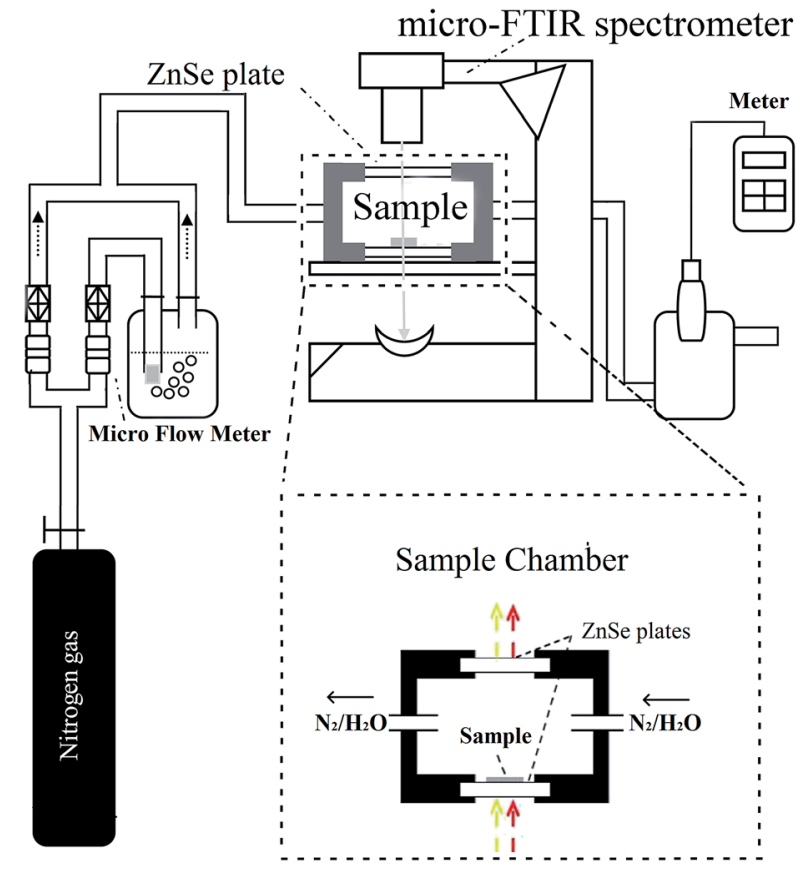

Fig. 1 The diagram of experimental apparatus used to study water adsorption of cellulose nanofiber film.

visual examinations and the selection of an observation area. ${ }^{36}$ This microscope was set to the optical mode, and the aperture was adjusted so as to be 100 by $100 \mu \mathrm{m}^{2}$; the desired observation areas for the cellulose nanofiber film were then defined. The micro-FTIR spectrum was collected using 64 scans between $720-4000 \mathrm{~cm}^{-1}$ using $4 \mathrm{~cm}^{-1}$ spectral resolutions. In order to obtain a higher signal-to-noise ratio, the spectra for cellulose nanofiber film and background were collected one after another in order to compensate for any moisture that may exist in the sample chamber.

Fig. 1 also shows a specially designed sample chamber. Once the cellulose nanofiber film was placed on the bottom of the chamber (which was composed of a ZnSe plate), the sample chamber was sealed by a lid (which contained another ZnSe plate). The ZnSe plates provided the optical paths for both the visible and IR light through this sample chamber. The RH in this chamber was accurately controlled using a stream of dry nitrogen and saturated vapor, whose ratio was changed using a high-precision gas micro-flow meter (Alicat Scientific, Tucson, $\mathrm{AZ}$, U.S.A.). The resulting $\mathrm{RH}$ was then measured using a humidity/temperature meter (Center 310, Center Technology Corp., New Taipei, Taiwan).

In the experiment, even if the $\mathrm{RH}$ of the chamber reaches the set value, a period of 60 min should be maintained. The equilibrium time was from a kinetic spectroscopic test. Before the start of this test, cellulose nanofiber film was equilibrated at the starting $\mathrm{RH}$, i.e., $0 \% \mathrm{RH}$, for $4 \mathrm{~h}$. We took the first spectrum measurement after starting, after which we changed the RH of the sample chamber to $5 \%$ and then recorded the spectra every 0.5 min. As shown in Fig. 2, after changing the target $\mathrm{RH}$ to a new setting, i.e., 5\% RH, there was typically a delay of approximately 3-4 min in which the actual $\mathrm{RH}$ approached the 


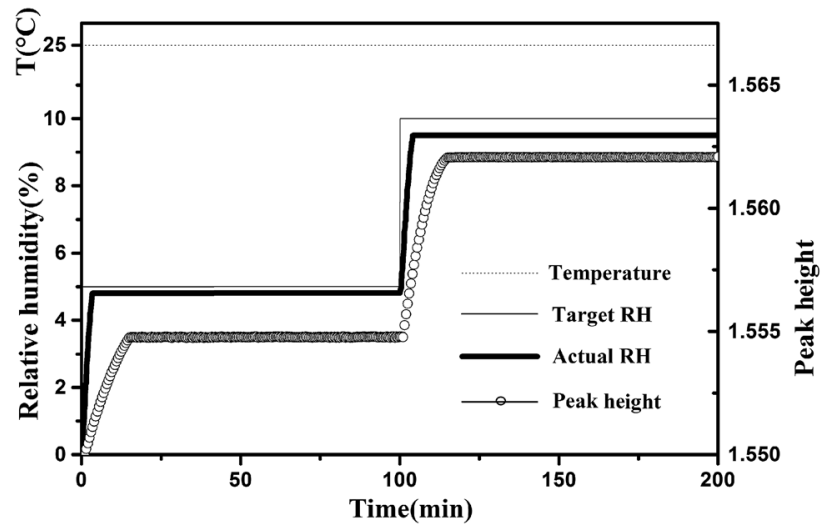

Fig. 2 Typical changes of target $\mathrm{RH}$, actual $\mathrm{RH}$ and peak height of the major peak at $3352 \mathrm{~cm}^{-1}$ during the water adsorption process. The temperature was maintained at $25^{\circ} \mathrm{C}$.

set target $\mathrm{RH}$. While, there were no changed detected in the measured spectra after a period of $15 \mathrm{~min}$, and this situation remained the same after $100 \mathrm{~min}$ (the peak height of the major peak at $3352 \mathrm{~cm}^{-1}$ was used to demonstrate the spectral change). And then the target $\mathrm{RH}$ was changed to next setting, i.e., $10 \% \mathrm{RH}$, no change was detected in the measured spectra after a period of $15 \mathrm{~min}$. In addition, the temperature in the specially designed sample chamber was maintained at $25{ }^{\circ} \mathrm{C}$. Based on these kinetic spectroscopy test results, we maintained a conditioning time of $60 \mathrm{~min}$ at each value prior to measurement to allow the sample to stabilize at the specific $\mathrm{RH}$.

\subsection{DVS apparatus}

A DVS apparatus (DVS AdvantagePlus, Surface Measurement Systems Ltd, London, U.K.) was used to determine the moisture content of cellulose nanofiber film. Data such as the run time, real-time masses of the samples, and $\mathrm{RH}$ at a constant temperature of $25{ }^{\circ} \mathrm{C}$ were obtained during the water sorption process. The RH was set to change from $0 \%$ to $95 \%$ in $5 \%$ steps and then decrease to $0 \%$. At every stage, the $\mathrm{RH}$ was kept constant for some time and then increased to the next increment as the sample mass decreased at $0.002 \% \min ^{-1}$ over a $10 \mathrm{~min}$ period. The moisture content was calculated using real-time mass measurements of the sample and the following equation:

$$
\mathrm{MC}=\frac{m_{1}-m_{2}}{m_{1}} \times 100 \%
$$

where, MC is the moisture content of the sample, $m_{1}$ is the dry mass of the sample, and $m_{2}$ is the real-time mass of the sample at a set RH.

In the experiment, typical changes of these data are shown in Fig. 3. After changing the target $\mathrm{RH}$ to a new setting, there was a delay of approximately 4-10 $\mathrm{min}$ in which the actual $\mathrm{RH}$ approached the set target RH. After the initial few minutes, the actual RH was kept stable, and then the fluctuation of the actual $\mathrm{RH}$ value was less than $0.1 \%$ at extended time. The real-time moisture content of the sample generated an asymptotic curve against time, and then reaches the equilibrium moisture

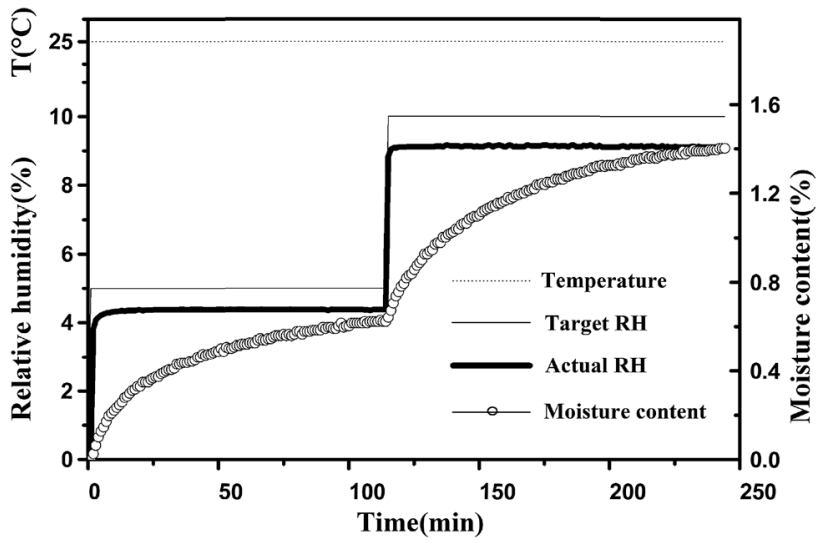

Fig. 3 Typical changes of target $\mathrm{RH}$, actual $\mathrm{RH}$ and moisture content during the water adsorption process. The temperature was maintained at $25^{\circ} \mathrm{C}$.

content. Meanwhile, it was found that the temperature values were very stable.

\subsection{Spectral data processing}

2.4.1 Obtaining difference spectra. In order to clearly demonstrate minor spectral changes during water adsorption process, ${ }^{37}$ difference spectra were obtained in the OMINIC 8.0 software by FTIR subtractive spectroscopy technique. By using this technique, difference spectra were acquired by subtracting the spectra at different $\mathrm{RH}$ values from the spectrum of dry cellulose nanofiber film in a $1: 1$ ratio.

2.4.2 Building quantitative prediction model. The quantitative prediction model based on micro-FTIR spectra was established by PLS-R method. In the process of establishing the model, the micro-FTIR spectra of cellulose nanofiber film in the $\mathrm{RH}$ range from $0 \%$ to $94 \%$ were all imported into TQ Analyst 9 (Thermo Scientific Inc., Madison, Wisconsin, USA). The type of optical path was set to "constant". The moisture content was defined as the only measured component, and the upper and lower values of the moisture content were set to $28 \%$ and $0 \%$, and the measured moisture contents using DVS apparatus were imported as reference values. No filters or no baseline corrections were used in the spectral preprocessing, and some regions of the original spectra associated with water adsorption were selected to construct model. Moreover, in order to avoid bias in the subset, the original spectra of cellulose nanofiber film at five replicates for each of $12 \mathrm{RH}$ levels (i.e., 60 spectra) were randomly selected into calibration groups, while five replicates per four other $\mathrm{RH}$ levels (i.e., 20 spectra) were selected as a validation group. The randomly selected spectra in the calibration group were used to perform calculations and crossvalidations; for this procedure, the best predictive model with the highest correlation of determination $\left(R^{2}\right)$ and lowest root mean square error of cross validation (RMSECV) was regressed after several iterations. Meanwhile, the spectra in the validation group were used for model evaluation. For this procedure, a predictive model was determined to be one with a high correlation of determination $\left(R^{2}\right)$ and a low root mean square error of prediction (RMSEP). 


\section{Results and discussion}

\subsection{Qualitatively analyzing the water adsorption of cellulose nanofiber film}

Fig. 4 shows the micro-FTIR spectra of cellulose nanofiber film at different $\mathrm{RH}$ levels during the water adsorption process. Meanwhile, the assignments of the main spectral peaks associated with water adsorption in the micro-FTIR spectrum of cellulose nanofiber film are summarized in Table $1 .^{\mathbf{3 8 - 4 0}}$

As shown in Fig. 4, the most intense peak at $3352 \mathrm{~cm}^{-1}$ was attributed to $\mathrm{O}-\mathrm{H}$ stretching vibration of cellulose nanofiber film and absorbed water, and it increased with a rise in $\mathrm{RH}$. The weaker peak at $1640 \mathrm{~cm}^{-1}$ was assigned to the $\mathrm{O}-\mathrm{H}$ bending vibration of adsorbed water, ${ }^{\mathbf{4 1}}$ and it was also observed to increase as the $\mathrm{RH}$ increased from 0 to $94 \%$. The spectral changes of these two peaks confirmed that $\mathrm{OH}$ groups were effective adsorption sites for adsorbed water. Moreover, the peak at $1160 \mathrm{~cm}^{-1}$ was assigned to that of $\mathrm{C}-\mathrm{O}-\mathrm{C}$ asymmetric stretching vibration at the $\beta$-glucosidic linkage, which was related to the cellulose chain. ${ }^{42}$ A continuous shift of this peak to lower wave numbers was observed as the RH increased. This shift was also caused by water adsorption, for absorbed water could stiffen the cellulose chain. ${ }^{\mathbf{4 3}}$ Based on these characteristics, it was found that the spectral regions affected by the water adsorption were $3700-3000 \mathrm{~cm}^{-1}, 1740-1618 \mathrm{~cm}^{-1}$, and $1190-$ $1139 \mathrm{~cm}^{-1}$.

Water adsorption in micro-FTIR spectroscopy provided unresolved broad peaks, which offered limited precise information. With the purpose of extracting minor spectral changes, difference spectra were obtained. Fig. 5 shows difference

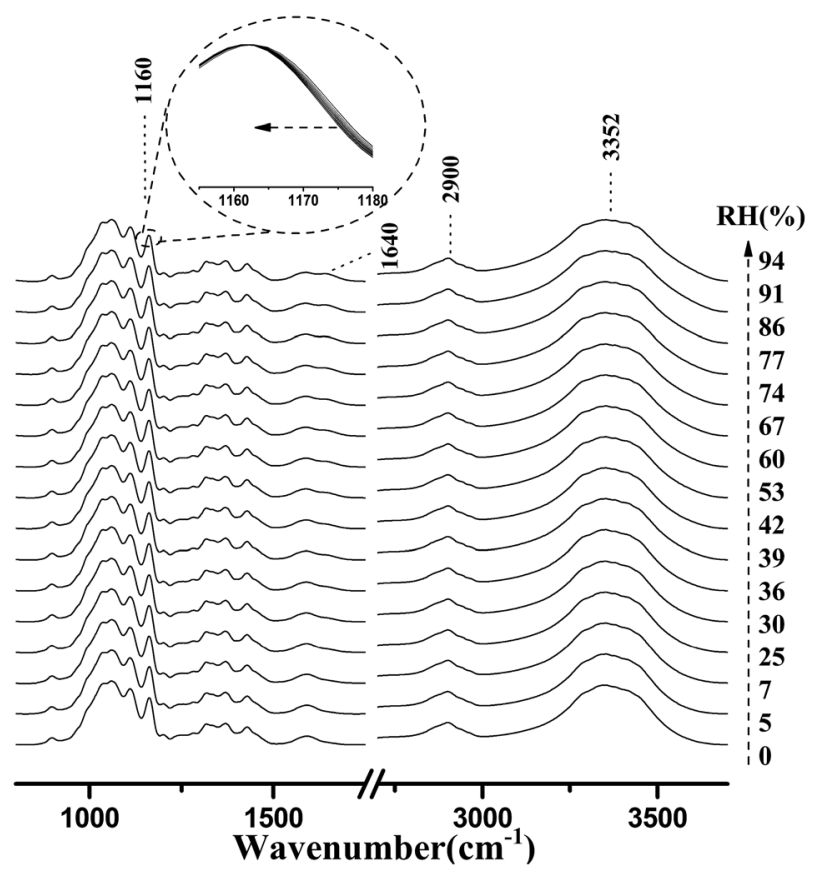

Fig. 4 Micro-FTIR spectra of cellulose nanofiber film during the water adsorption process. The arrow demonstrated that the $\mathrm{RH}$ values were increased from $0 \%$ to $94 \%$.
Table 1 The assignments of the main spectral peaks associated with water adsorption in the micro-FTIR spectrum of cellulose nanofiber film

\begin{tabular}{ll} 
Wave number $\left(\mathrm{cm}^{-1}\right)$ & Assignment \\
\hline $3700-3000$ & O-H stretching vibration \\
$3000-2700$ & C-H stretching vibration \\
1640 & O-H bending vibration of adsorbed water \\
1163 & C-O-C asymmetric stretching vibration \\
& at $\beta$-glucosidic linkage
\end{tabular}

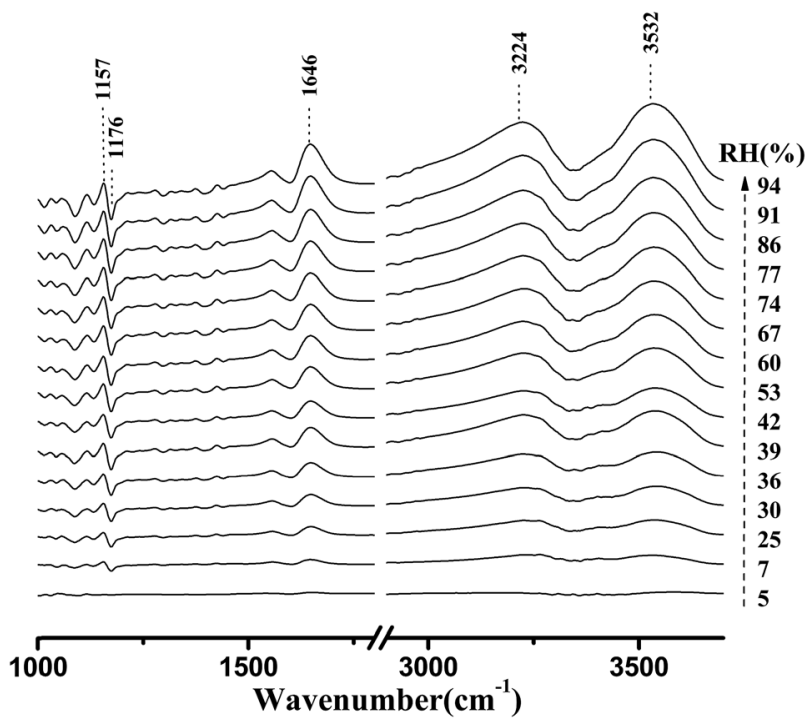

Fig. 5 Difference micro-FTIR spectra of cellulose nanofiber film during the water adsorption process. The arrow demonstrated that the $\mathrm{RH}$ values were increased from $5 \%$ to $94 \%$.

spectra of cellulose nanofiber film at various RH levels from 5 to $94 \%$. The signature of the main peak in the spectral region of $3700-2852 \mathrm{~cm}^{-1}$ increased which was identified and assigned to absorbed water. Similar growth trends of this broad peak had been found in other measurements of cellulose materials. ${ }^{44}$ It can be concluded that the whole spectral region of 3700$2852 \mathrm{~cm}^{-1}$ was related to the water adsorption. Meanwhile, the positive-going peak at $1646 \mathrm{~cm}^{-1}$ changed continuously whilst the RH increased. The intensity of the peak at $1176 \mathrm{~cm}^{-1}$ was observed to decrease with an increase in $\mathrm{RH}$, while the signature from the peak at $1157 \mathrm{~cm}^{-1}$ was observed to rise. The changes of these three peaks further indicated that the spectral regions of 1740-1618 $\mathrm{cm}^{-1}$, and $1190-1139 \mathrm{~cm}^{-1}$ were affected by the water adsorption. In summary, the study results demonstrated that the regions of micro-FTIR spectra closely associated with water adsorption were $3700-2852 \mathrm{~cm}^{-1}, 1740-1618 \mathrm{~cm}^{-1}$, and 1190-1139 $\mathrm{cm}^{-1}$.

Fig. 6 shows the development of the peak height as a function of $\mathrm{RH}$, for these three major peaks affected by the water adsorption. As shown in this figure, these three major peaks had different growth rates. The discrepancies were observed in many cellulose materials. Moreover, a univariate analysis of 


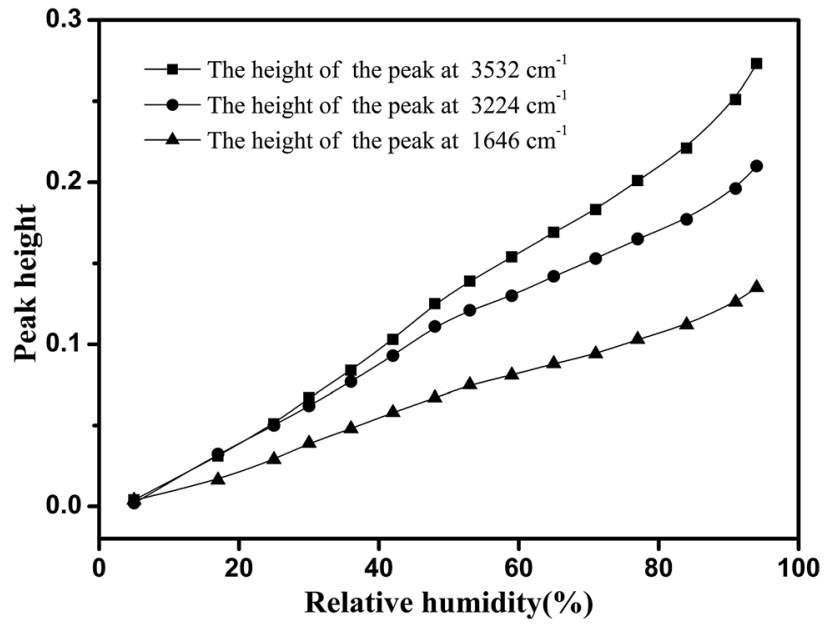

Fig. 6 Peak height of three major peaks associated with water adsorption taken from difference spectra over the set $\mathrm{RH}$ range. Quadrate dot: the height of the peak at $3532 \mathrm{~cm}^{-1}$. Circular dot: the height of the peak at $3224 \mathrm{~cm}^{-1}$. Triangle dot: the height of the peak at $1646 \mathrm{~cm}^{-1}$.

these peaks failed to properly reproduce the sorption isotherms. ${ }^{29}$ Therefore, a multivariate approach, such as a PLS$\mathrm{R}$ method, should be introduced to qualitatively characterize the water adsorption.

\subsection{Quantitative analysis of the water adsorption in cellulose nanofiber film}

3.2.1 Moisture contents of cellulose nanofiber measured by DVS. As mentioned in Section 1, DVS can be used as a reference method to quantitatively analyze water adsorption. Here, using this apparatus, moisture contents of cellulose nanofiber film were obtained as reference values. Fig. 7a shows the moisture content of cellulose nanofiber film with the varying $\mathrm{RH}$ levels over the time profile in the isotherm run. In this figure, the RH was changed step-by-step and the sorption kinetics was recorded. Based on this, by plotting the respective equilibrium moisture contents versus the corresponding level of $\mathrm{RH}$, sorption isotherm was obtained as shown in Fig. 7b.

3.2.2 Development of the PLS-R quantitative models. As mentioned in Section 2.4.2, the spectral regions are important parameters for constructing a PLS-R quantitative model. Here, three spectral regions of $3700-2852 \mathrm{~cm}^{-1}, 1740-1618 \mathrm{~cm}^{-1}$, and 1190-1139 $\mathrm{cm}^{-1}$ affected by water adsorption were used to construct the quantitative prediction model as one proposed case. On this basis, the increase and decrease of these three spectral regions were also introduced as the other two cases for comparison (3700-2852 $\mathrm{cm}^{-1}, 2852-2538 \mathrm{~cm}^{-1}, \quad 1740-$ $1618 \mathrm{~cm}^{-1}$, and $1190-1139 \mathrm{~cm}^{-1}$, second case; $3700-3000 \mathrm{~cm}^{-1}$, $2852-2538 \mathrm{~cm}^{-1}, 1740-1618 \mathrm{~cm}^{-1}$, and $1190-1139 \mathrm{~cm}^{-1}$, third case). These three cases and their corresponding spectral regions, RESECV, RESEP, and $R^{2}$ values in the PLS-R models are all summarized in Table 2 . These PLS-R quality parameters such as RESECV, RESEP, and $R^{2}$ were described before. ${ }^{45}$ The first model using three spectral regions of $3700-2852 \mathrm{~cm}^{-1}, 1740-$
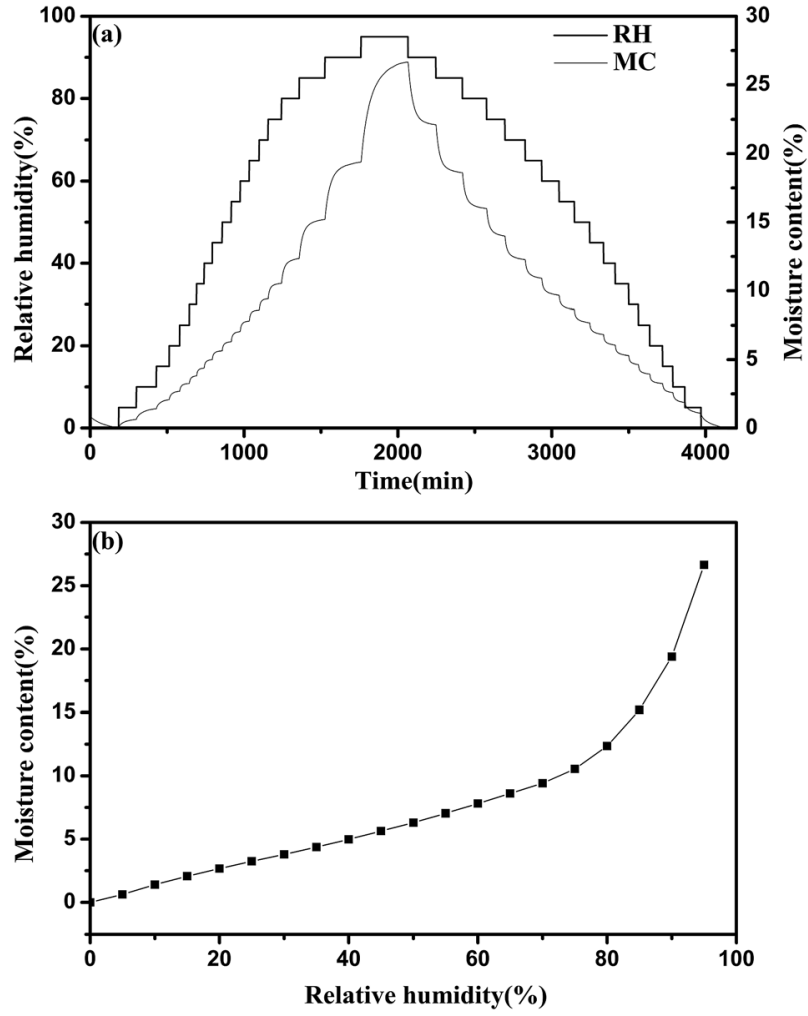

Fig. 7 (a) The moisture content of cellulose nanofiber film with the varying $\mathrm{RH}$ levels over the time profile in the isotherm run. (b) The sorption isotherm of cellulose nanofiber film measured by DVS apparatus.

$1618 \mathrm{~cm}^{-1}$, and $1190-1139 \mathrm{~cm}^{-1}$ had the highest coefficients of determination, lowest RMSEP and RMSECV values which showed that this model had good estimated performance and high prediction accuracy. What's more, these results suggested that the best model was one which used complete spectral regions closely associated with water adsorption, and the increase or decrease of these spectral regions would lower the quality of the quantitative model.

With the help of this optimal PLS-R quantitative model, we estimated the moisture contents of cellulose nanofiber film using the micro-FTIR spectra collected over the entire $\mathrm{RH}$ range of $0-94 \%$. The calculated moisture contents of cellulose nanofiber film in the full set $\mathrm{RH}$ range during the adsorption process in the isothermal sorption run are shown in Fig. 8. This sorption isotherm exhibited a typical sigmoidal-shaped curve in the adsorption isotherm run; this has been observed in other cellulose materials. ${ }^{\mathbf{4 6 , 4 7}}$ The water adsorption process of cellulose nanofiber film can also be separated into three stages, as explained in previous studies. ${ }^{48}$ In the first stage $(\mathrm{RH}<35 \%)$, the absorbed water is bound directly to the chemical adsorption sites through hydrogen bonding, and is related to Langmuir's mode. In the second stage $(35 \%<\mathrm{RH}<55 \%)$, the absorbed water is indirectly bonded to hydrophilic groups through the absorbed water, and the water content increases with $\mathrm{RH}$ as per Henry's model. In the third section ( $\mathrm{RH}>55 \%)$, the absorbed water interacts with them, and forms a five-molecule 
Table 2 PLS-R quality parameters for cross and test set validation for the proposed three cases $^{a}$

\begin{tabular}{llll}
\hline & First case & Second case & Third case \\
\hline Spectral & $3700-2852 /$ & $3700-2852 /$ & $3700-3000 /$ \\
region $\left(\mathrm{cm}^{-1}\right)$ & $1740-1618 /$ & $2852-2538 /$ & $1740-1618 /$ \\
& $1190-1139$ & $1740-1618 /$ & $1190-1139$ \\
& & $1190-1139$ &
\end{tabular}

Cross validation RMSECV

Number of PLS

components

$R^{2}(\%)$

$$
\begin{aligned}
& 0.637 \\
& 6
\end{aligned}
$$

99.66

0.882

6

0.658

6

99.35

99.62

External validation

\section{RMSEP}

Number of PLS

components

$R^{2}(\%)$

$\begin{array}{lll}0.439 & 0.561 & 0.530 \\ 6 & 6 & 6 \\ 99.52 & 99.29 & 98.94\end{array}$

${ }^{a}$ RMSECV, root mean square error of cross validation; RMSEP, root mean square error of prediction; $R^{2}$, coefficient of determination.

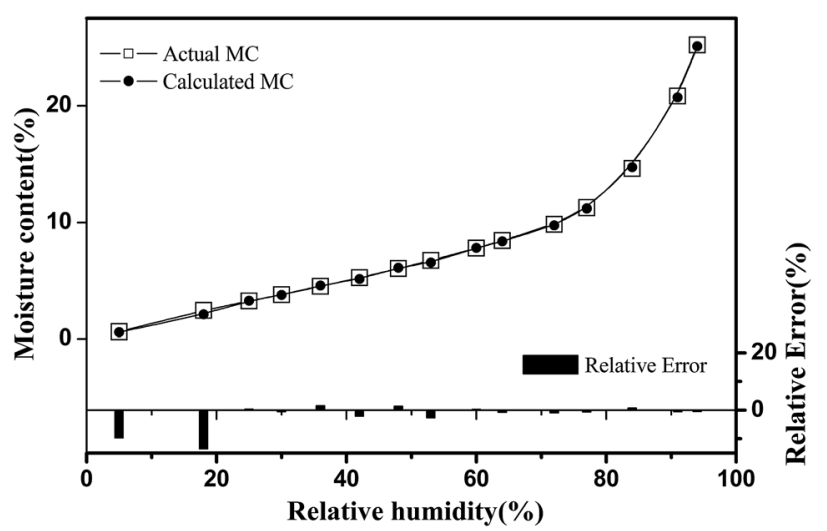

Fig. 8 Comparison of the sorption isotherms of cellulose nanofiber film calculated by the PLS-R quantitative model and measured by DVS approaches. Quadrate dot: the moisture content measured by DVS apparatus. Circular dot: the calculated moisture content using PLS-R model.

tetrahedral structure. As shown in Fig. 8, the predicted values and reference values were very similar in the $\mathrm{RH}$ region of 35-94\% (relative error was lower than 3\%), and it confirmed the effectiveness of micro-FTIR spectroscopy in characterizing water adsorption of cellulose nanofiber film. Meanwhile, the relative errors were different in different stages of the water adsorption process, and this difference may be due to abovementioned adsorption mechanism.

\section{Conclusions}

By using in situ micro-FTIR spectroscopy, a detailed investigation on water adsorption of cellulose nanofiber film was achieved. As the RH increased from $0 \%$ to $94 \%$, the increase of the characteristic peaks at $3352 \mathrm{~cm}^{-1}$ and $1604 \mathrm{~cm}^{-1}$ indicated that
$\mathrm{OH}$ groups were effective adsorption sites for adsorbed water. Meanwhile, the difference between the moist and dry spectra identified that three spectral regions of $3700-2852 \mathrm{~cm}^{-1}, 1740-$ $1618 \mathrm{~cm}^{-1}$, and $1190-1139 \mathrm{~cm}^{-1}$ were closely related to the water adsorption. On this basis, using PLS-R method, three multivariate models linking moisture contents and the identified micro-FTIR spectral regions were developed. By comparing these three models, it was found that the best model was one which used complete spectral regions closely associated with water adsorption. Furthermore, using this optimal PLS-R quantitative model, water sorption isotherm was determined, and it showed that the predicted moisture contents were well consistent with the reference values obtained using DVS apparatus in the RH region of 35-94\% (relative error was lower than $3 \%$ ). This study confirmed that the effectiveness of micro-FTIR spectroscopy in characterizing water adsorption of cellulose nanofiber film at room temperature of $25^{\circ} \mathrm{C}$, which could assist in developing a non-destructive and rapid method for qualitatively and quantitatively analyzing water adsorption of nanocellulose materials.

\section{Conflicts of interest}

There are no conflicts to declare.

\section{Acknowledgements}

Research is financially supported by National Natural Science Foundation of China (Grant No. 31500475 and 31530009), China Postdoctoral Science Foundation (Grant No. 2014M562146 and 2016T90768), Research Foundation of Education Bureau of Hunan Province (Grant No. 15B256) and Hunan Province Science and Technology Project (Grant No. 2015RS4046).

\section{References}

1 L. Berglund, M. Noël, Y. Aitomäki, T. Öman and K. Oksman, Ind. Crops Prod., 2016, 92, 84-92.

2 M. L. Hassan, A. P. Mathew, E. A. Hassan, N. A. El-Wakil and K. Oksman, Wood Sci. Technol., 2010, 46, 193-205.

3 M. F. Rosa, E. S. Medeiros, J. A. Malmonge, K. S. Gregorski, D. F. Wood, L. H. C. Mattoso, G. Glenn, W. J. Orts and S. H. Imam, Carbohydr. Polym., 2010, 81, 83-92.

4 C. Liu, Z. Shao, J. Wang, C. Lu and Z. Wang, RSC Adv., 2016, 6, 97912-97920.

5 Z. Xu, J. Li, H. Zhou, X. Jiang, C. Yang, F. Wang, Y. Pan, N. Li, X. Li, L. Shi and X. Shi, RSC Adv., 2016, 6, 43626-43633.

6 N. Manhas, K. Balasubramanian, P. Prajith, P. Rule and S. Nimje, RSC Adv., 2015, 5, 23999-24008.

7 X. Shi, Y. Zheng, G. Wang, Q. Lin and J. Fan, $R S C A d v .$, 2014, 4, 47056-47065.

8 B. D. Vogt, C. L. Soles, H. J. Lee, E. K. Lin and W. L. Wu, Polymer, 2005, 46, 1635-1642.

9 A. Isa, J. Minamino, H. Mizuno, S. Suzuki, Y. Kojima, H. Ito, R. Makise, M. Okamoto and T. Hasegawa, J. Wood Chem. Technol., 2013, 33, 208-216. 
10 B. D. Vogt, C. L. Soles, H. Lee, E. K. Lin and W. Wu, Langmuir, 2004, 20, 1453-1458.

11 S. Belbekhouche, J. Bras, G. Siqueira, C. Chappey, L. Lebrun, B. Khelifi, S. Marais and A. Dufresne, Carbohydr. Polym., 2011, 83, 1740-1748.

12 Y. Xie, C. A. S. Hill, Z. Jalaludin and D. Sun, Cellulose, 2011, 18, 517-530.

13 H. Sugimoto, T. Miki, K. Kanayama and M. Norimoto, J. NonCryst. Solids, 2008, 354, 3220-3224.

14 A. Célino, O. Goncalves, F. Jacquemin and S. Freour, Carbohydr. Polym., 2014, 101, 163-170.

15 Y. B. Grunin, L. Y. Grunin, D. S. Masas, V. I. Talantsev and N. N. Sheveleva, Russ. J. Phys. Chem. A, 2016, 90, 2249-2253.

16 R. H. Newman and T. C. Davidson, Cellulose, 2004, 11, 23-32. 17 Y. Xie, C. A. S. Hill, Z. Jalaludin, S. F. Curling, R. D. Anandjiwala, A. J. Norton and G. Newman, J. Mater. Sci., 2011, 46, 479-489.

18 Y. Xie, C. A. S. Hill, Z. Jalaludin and D. Sun, Cellulose, 2011, 18, 517-530.

19 C. A. S. Hill, B. Keating, K. Laine, L. Rautkari, M. Hughes and B. Constant, J. Mater. Sci., 2012, 47, 3191-3197.

20 R. Kohler, R. Dück, B. Ausperger and R. Alex, Compos. Interfaces, 2003, 10, 255-276.

21 C. A. S. Hill, A. Norton and G. Newman, J. Appl. Polym. Sci., 2010, 116, 2166-2173.

22 S. Okubayashi, U. J. Griesser and T. Bechtold, Cellulose, 2005, 12, 403-410.

23 K. Kachrimanis, M. F. Noisternig, U. J. Griesser and S. Malamataris, Eur. J. Pharm. Biopharm., 2006, 64, 307-315.

24 P. S. Madamba, R. H. Driscoll and K. A. Buckle, J. Food Eng., 1996, 29, 75-97.

25 M. J. Lundahl, A. G. Cunha, E. Rojo, A. C. Papageorgiou, L. Rautkari, J. C. Arboleda and O. J. Rojas, Sci. Rep., 2016, 6, 30695.

26 C. A. S. Hill, A. Norton and G. Newman, Holzforschung, 2010, 64, 469-473.

27 C. A. S. Hill, A. Norton and G. Newman, J. Appl. Polym. Sci., 2009, 112, 1524-1537.
28 K. Wehbe, J. Filik, M. Frogley and G. Cinque, Anal. Bioanal. Chem., 2013, 405, 1311-1324.

29 A. Olsson and L. Salmen, Carbohydr. Res., 2004, 339, 813818.

30 K. Haxaire, Y. Maréchal, M. Milas and M. Rinaudo, Biopolymers, 2003, 72, 10-20.

31 K. Hofstetter, B. Hinterstoisser and L. Salmén, Cellulose, 2006, 13, 131-145.

32 K. Kachrimanis, M. F. Noisternig, U. J. Griesser and S. Malamataris, Eur. J. Pharm. Biopharm., 2006, 64, 307-315.

33 J. Berthold, R. O. Olsson and L. Salmén, Cellulose, 1998, 5, 281-298.

34 X. Guo, Y. Wu and N. Yan, Holzforschung, 2017, 71, 415-423.

35 J. Han, C. Zhou, Y. Wu, F. Liu and Q. Wu, Biomacromolecules, 2013, 14, 1529-1540.

36 Y. Liu, Z. Yang, Y. Desyaterik, P. L. Gassman, H. Wang and A. Laskin, Anal. Chem., 2008, 80, 633-642.

37 J. Guo, K. Song, L. Salmen and Y. Yin, Carbohydr. Polym., 2015, 115, 207-214.

38 I. M. D. Rosa, J. M. Kenny, D. Puglia, C. Santulli and F. Sarasini, Compos. Sci. Technol., 2010, 70, 116-122.

39 N. Abidi, L. Cabrales and C. H. Haigler, Carbohydr. Polym., 2014, 100, 9-16.

40 C. Y. Liang and R. H. Marchessault, J. Appl. Polym. Sci., 1959, 39, 269-278.

41 T. Wandlowski, K. Ataka, S. Pronkin and D. Diesing, Electrochim. Acta, 2004, 49, 1233-1247.

42 M. Åkerholm and L. Salmén, Polymer, 2001, 42, 963-969.

43 K. Tashiro and M. Kobayashi, Polymer, 1991, 32, 1516-1526.

44 X. Guo, Y. Qing, Y. Wu and Q. Wu, Int. J. Biol. Macromol., 2016, 83, 117-125.

45 M. Fitzpatrick, P. Champagne and M. F. Cunningham, Carbohydr. Polym., 2012, 87, 1124-1130.

46 S. Alix, E. Philippe, A. Bessadok, L. Lebrun, C. Morvan and S. Marais, Bioresour. Technol., 2009, 100, 4742-4749.

47 F. Gouanvé, S. Marais, A. Bessadok, D. Langevin and M. Métayer, Eur. Polym. J., 2007, 43, 586-598.

48 X. Guo and Y. Wu, J. Wood Chem. Technol., 2017, 37, 383-392. 